\title{
High refractive index films of polymer nanocomposites
}

\author{
Lorenz Zimmermann, Martin Weibel, Walter Caseri, and Ulrich W. Suter \\ Eidgenössische Technische Hochschule, Institut für Polymere, ETH-Zentrum, CH-8092 Zürich, \\ Switzerland
}

(Received 6 July 1992; accepted 19 .March 1993)

Solutions of $\mathrm{PbS}$ particles and gelatin were uscd for the preparation of nanocomposites by a spin-coating process. This allows for the preparation of nanocomposite films with controlled thickness, c.g., between $40 \mathrm{~nm}$ and $2 \mu \mathrm{m}$ for a film containing $45 \mathrm{wt}$. $\%$ PbS. Surface roughness and film thickness were investigated by surface profilometry and scanning electron microscopy (SEM). The refractive index at $632.8 \mathrm{~nm}$ can be expressed by a linear function of the volume fraction of $\mathrm{PbS}$ in the range of 0 to $55 \mathrm{vol} . \%$ $\mathrm{PbS}$. In this range, the refractive index increases from 1.5 to 2.5 with increasing $\mathrm{PbS}$ ratio and belongs, therefore, to the highest refractive indices known for polymeric composite materials.

\section{INTRODUCTION}

Composites between polymers and inorganic particles in the micrometer range are often opaque. ${ }^{1,2}$ Light scattering, responsible for the opacity, can be suppressed cither by using materials with nearly matching refractive indices ${ }^{3}$ or by decreasing the filler's dimensions to a range below ca. $50 \mathrm{~nm} .{ }^{1.2 .45}$ Therefore, nanocomposites of polymers and inorganic colloids can act as optically homogeneous materials with modified optical propertics. ${ }^{1,2,4,6,7}$ Nanocomposites containing lead sulfide, for instance, can act as optical filters, ${ }^{+}$nonlinear optical," or "ultrahigh" refractive index materials."

The refractive index of polymers is usually between 1.3 and 1.7. Examples of higher refractive index are, e.g., 2.12 for poly(thiophenc) $)^{y}$ and 1.7245 for $\left[\mathrm{CH}_{2-}\right.$ $\left.\mathrm{CH}=\mathrm{CHCH}_{2} \mathrm{~N}^{+}\left(\mathrm{CH}_{2} \mathrm{CH}_{3}\right)_{2}\left(\mathrm{CH}_{2}\right)_{6} \mathrm{~N}^{-}\left(\mathrm{CH}_{2} \mathrm{CH}_{3}\right)_{2}\right]_{n}$. $n$ - $\mathrm{HgI}_{4}^{2-} \cdot{ }^{10}$ Aromatic polyamides can exhibit (isotropic) refractive indices up to $2.05^{11}$ or $2.07 .^{12}$

The refractive index of polymers can be enhanced by the addition of colloidal particles with high refractive index, ${ }^{7}$ e.g., $\mathrm{PbS}$ that exhibits (at least in crystalline form) a refractive index on the order of 4 in a wide wavelength range. ${ }^{13}$ The highest refractive index for polymeric materials, i.e., pure polymers or polymer composites, has been obtaincd to date, to our best knowledge, for nanocomposiles of lead sulfide and poly(cthyleneoxide). ${ }^{7}$ They exhibit refractive indices of up to 2.9 at $632.8 \mathrm{~nm}$ and of up to $3.0-3.4$ in the whole range of $1000-2500 \mathrm{~nm}$. These nanocomposites were prepared by adding a hydrogen sulfide solution to a solution containing the polymer and $\mathrm{Pb}$ (II) ions. The genesis of colloidal $\mathrm{PbS}$ particles and the precipitation of the nanocomposite material take place simultancously and immediately after addition of hydrogen sulfide. This method allows for the preparation of nanocomposites filled to ca. 50) vol. \% PbS; however, attempts to prepare nanocomposites with a markedly lower PbS-content, e.g., by variation of the initial $\mathrm{Pb}^{2+} /$ poly(ethyleneoxide) ratio, failed. Also, experiments aimed at preparing nanocomposite films were inconclusive because of poor film quality.

In this report, we describe the preparation of polymer nanocomposite films with controlled layer thickness and refractive index, starting from homogeneous solutions containing colloidal PbS and a polymer.

\section{EXPERIMENTAL}

\section{A. Chemical reagents}

Gelatin (from pork skin, high gel strength) and lead acelate were obtained from Fluka (Buchs, Switzerland), hydrogen sulfide from Pangas (Lucerne, Switzerland). From gelatin, the following values were obtained by microanalysis: $\mathrm{C}, 43.41 \%, \mathrm{H}, 6.64 \%, \mathrm{~N}, 16.25 \%$, and $\mathrm{H}_{2} \mathrm{O}, 5.20 \%$.

\section{B. Preparation of nanocomposite films}

Hydrogen sulfide solutions were prepared as follows: In a gas wash flask, nitrogen was bubbled for some minutes through ca. $30 \mathrm{ml}$ water under stirring to remove some dissolved oxygen. Then, hydrogen sulfide gas was bubbled through the gas wash flask for $15-30 \mathrm{~min}$; excess hydrogen sulfide was absorbed in sodium hydroxide solution. The concentration of such a hydrogen sulfide solution is $0.04-0.08 \mathrm{M}$, determined by back-titration with hydrochloric acid. ${ }^{\text {' Higher con- }}$ centrations (up to $(1.17 \mathrm{M}$ ) were obtained by cooling the solution during the introduction of the hydrogen sulfide to ca. $5^{\circ} \mathrm{C}$. 
For the preparation of films with low PbS content (bclow 40 wt. \%), gelatin (typically ca. $30 \mathrm{mg}$ ) was dissolved in water (typically ca. $1 \mathrm{ml}$ ) at $50-60{ }^{\circ} \mathrm{C}$, and lead acetate (the amount corresponding to the desired final concentration of $\mathrm{PbS}$ in the film) was added after the gelatin was dissolved. For the preparation of films with high $\mathrm{PbS}$ content, gelatin (typically $10-50 \mathrm{mg}$ ) was dissolved in a $0.1 \mathrm{M}$ lead acetate solution (typically $1.3-2.5 \mathrm{ml}$ ) at $50-60{ }^{\circ} \mathrm{C}$. Then, in both cases, the solution was stirred well and cooled to room temperature. Under vigorous stirring hydrogen sulfide solution was added as fast as possible (ca. 1\% stoichiometric excess with respect to lead, the solution being at room temperature or at $5^{\circ} \mathrm{C}$, as indicated by the necessary hydrogen sulfide concentration). For the preparation of films with more than ca. $40 \mathrm{wt} . \% \mathrm{PbS}$, hydrogen sulfide concentrations of $0.09-0.17 \mathrm{M}$ were used in order to keep the total volume of the solution low. Uniform stirring is important since otherwise coagulation can occur, especially for high $\mathrm{PbS} /$ gelatin ratios. After complete mixing the solutions were allowed to stand for $1 \mathrm{~h}$. During this time, gelation increased the viscosity to suitable values for the spin-coating process.

Spin coating was performed on a CONVAC: $1001 \mathrm{~S} /$ ST 147 using glass substrates, if not otherwise indicated in the text. About three drops of the solution were spread over an arca of $\mathrm{ca} .10 \mathrm{~cm}^{2}$ with a spatula at a spinning rate of $50 \mathrm{rpm}$ (the exact amount of liquid is not critical with respect to the final layer thickness). Thereafter, the spinning rate was increased to $100-4000 \mathrm{rpm}$, depending on the viscosity of the solution and the required layer thickness. After rotation for $30-90 \mathrm{~s}$, the spinning process was interrupted and started again at $150 \mathrm{rpm}$ for $10 \mathrm{~s}$.

The films then were dried at a pressure of ca. 100 Torr in a slight and constant stream of air. It is important that the film is exactly horizontal for films with high regularity. The stream of air was moistened in order to prevent fissures in the final film. This drying procedure was carried out, e.g., during $2 \mathrm{~h}$ for a film thickness of $0.2 \mu \mathrm{m}$ and 2 days for a film of $30 \mu \mathrm{m}$.

Films designated in the text as "annealed films" were subsequently held at $164^{\circ} \mathrm{C}$ and 100 Torr for $2 \mathrm{~h}$.

\section{Chemical analysis of the nanocomposites}

Elemental analysis for carbon and determination of the water content were carried out by Dieter Manser of the Microanalytic Laboratory Service of the Institut für organische Chemic, ETH-Zürich.

Microanalysis of a film containing $40 \mathrm{wt} \% \mathrm{PbS}$ (calculated on the basis of the known $\mathrm{PbS} /$ gelatin ratio in the solution used for the film preparation) yielded the following values (in parentheses the calculated values based on the analysis of the pure gelatin, see A. Chemical
Reagents): C, $26.53 \%(26.05 \%), \mathrm{H}, 3.88 \%(3.98 \%), \mathrm{N}$, $9.75 \%(9.25 \%)$, and $\mathrm{H}_{2} \mathrm{O}, 2.45 \%(3.12 \%)$. This shows in particular that the water/gelatin ratio in the films is comparable to the ratio in "native" gelatin. Further, it was found that the annealing procedure does not reduce the water content significantly.

\section{Surface profilometry}

Surface profiles. surface roughnesses, and film thicknesses werc detcrmined by an Alphastep 200 Surface Profilometer (Tencor Instruments) with a stylus of radius $1.5-2.5 \mu \mathrm{m}$. The stylus force was adjusted to $9 \mathrm{mg}$, and a scan time of $40 \mathrm{~s}$ was selected. The surface roughness is calculated as the average deviation in the height of the profile from the mean level. For film thickness measurements, an area of the film was selected that appeared homogeneous to the eye. In this area, a small part of the film was removed with a spatula, creating an artificial step at which the film thickness was determined.

\section{E. Refractive index measurements}

Refraclive index measurements were performed on a PLASMOS SD 2300 ellipsometer equipped with a $\mathrm{He}-\mathrm{Ne}$ laser $(\lambda=632.8 \mathrm{~nm})$ at an angle of incidence of $70^{\circ}$. Refractive indices were measured at 10 separate spots, and 5 measurements were performed at each spot resulting in a total of 50 measurements per sample. In the text, the corresponding average value is indicated.

To be sure that the measured refractive index is not influenced by interference effects, i.e., reflections of a part of the incident beam on the surface of the substrate (c.g., glass), films of different thicknesses were investigated. An example follows: Films containing 45 wt. \% PhS were prepared on glass substrates (refractive index $1.423 \pm 0.040,95 \%$ confidence level) in a thickness range between $40 \mathrm{~nm}$ and $2 \mu \mathrm{m}$. Between $700 \mathrm{~nm}$ and $2 \mu \mathrm{m}$ thickness, these films show the brown color of colloidal lead sulfide. Their refractive index does not depend on thickness $(1.807 \pm 0.027,95 \%$ confidence level). To verify that there is no influence of the substrate, anncaled films of 1 and $2 \mu \mathrm{m}$ were prepared on silicon wafers (refractive index $3.8071 \pm 0.0024,95 \%$ confidence level). Their color is also brown, and the measured refractive indices are 1.818 and 1.802 . On glass, refractive indices in the same range are also found for films with a thickness between 40 and $250 \mathrm{~nm}$. They also exhibit a brown color. However, between 250 and $700 \mathrm{~nm}$, the films show interference colors different from brown. ${ }^{14,15}$ In this thickness region, no consistent indices could be deduced from the mcasurements.

\section{F. Electron microscopy}

For transmission electron microscopy, a gelatin capsule (diameter and height ca. $5 \mathrm{~mm}$ ) was filled with 
epon-araldite, ${ }^{16}$ turned upside down upon the nanocomposite film (on glass), and cured at $60^{\circ} \mathrm{C}$ for $24 \mathrm{~h}$. After cooling to room temperature, the sample was plunged into liquid nitrogen whereupon the polymer components detached from the support. The material was cut in pieces of ca. $4 \mathrm{~mm}^{2}$ area and ca. $0.5 \mathrm{~mm}$ thickness and embedded again in epon-araldite. After hardening as described above, thin sections (interference color silver) were cut on a Reichert Ultracut Microtome with a diamond knife (Diatome, Bicl-Bienne, Switzerland). Sections were floated on the water as trough liquid. The electron micrographs were taken on a Philips EM 301 with an acceleration voltage of $80 \mathrm{kV}$.

The SEM (scanning electron microscopy) samples were coated with $3 \mathrm{~nm}$ platinum-carbon by electron beam evaporation in a BAF 120 (Balzers) device. Specimens were investigated in a Hitachi $S-900$ in-lens field emission SEM using the secondary electron signal.

\section{RESULTS AND DISCUSSION}

\section{A. Preparation and characterization of films}

Highly filled PbS-polymer nanocomposites can be prepared from homogeneous solutions containing colloidal $\mathrm{PbS}$ and polymer (this is favored over exposure to hydrogen sulfide of a film containing $\mathrm{Pb}^{2+7}$ ). Among several water-soluble polymers tested, i.c., poly(vinyl alcohol), poly(acrylamide), poly(acrylic acid), poly(ethyleneoxide), and gelatin, the most suitable was found to be gelatin.

The homogeneous polymer-PbS solutions can be used for the preparation of films. The simplest method for this purpose is to evaporate the solvent from a stagnant "puddle", either at ambient conditions, at clevated temperature, or at reduced pressure. This allowed for the preparation of homogeneous films with a $\mathrm{PbS}$ content of up to ca. $65 \mathrm{wt} \% \mathrm{PbS}$. It is also possible to prepare homogeneous solutions with $\mathrm{PbS}$ /gelatin ratios corresponding to higher PbS contents in the film; however, local phase separation occurs upon solvent evaporation if the $\mathrm{PbS}$ /gelatin ratio is too high. Moreover, the films manufactured by this simple solvent evaporation are not uniform (the film thickness decreases with increasing distance from the center of the film).

Another method for the preparation of polymer ${ }^{15,17-21}$ or polymer-dye ${ }^{22}$ films is spin coating. We found that homogeneous PbS-gelatin films can be created by spin coating with higher $\mathrm{PbS}$ content than is possible with the other methods investigated. Uniform films could be obtained when the substrate is initially covered with the $\mathrm{PbS}$-gelatin solution over the entire area where the film is finally desired (here typically $10 \mathrm{~cm}^{2}$ ); upon spinning $(100-4000 \mathrm{rpm})$, the area originally covered with solution yields an even film, while outside of this area stripes are formed. In this way, it was possible to produce films with a PbS content of up to 86.4 wt. \%. However, films with a PbS content below ca. 70 wt. \% were of significantly better quality than those containing more $\mathrm{PbS}$. The films show the brown color of colloidal PbS.

The viscosity of gelatin or gelatin-PbS solutions can be controlled by the concentration and the age of the solution, i.e., the degree of gelation. Good conditions for spin coating were found for a gelation time of $1 \mathrm{~h}$.

Depending on the thickness and PbS content, a portion of light passes through the films, and this light is not scattered as far as can be judged by the cye. It has been suggested that the refractive index of the two components must be closely matched, in nanocomposites, to avoid cloudiness. ${ }^{1,2}$ Our result on PbS-polymer nanocomposites agrees with Mahler's conclusion ${ }^{4,5}$ that this aspect seems to be of little importance, provided that the particle size as well as the interparticle distances are much smaller than the wavelength of light. The absorption spectrum of the $\mathrm{PbS}$ films corresponds to that of colloidal $\mathrm{PbS}$ with a particle diameter of ca. 5-20 nm. ${ }^{23}$

Thermogravimetry (TGA) of a film with a $\mathrm{PbS}$ content of $66.6 \mathrm{wt} \%$ shows that the thermal stability of the composite is comparable with that of "native" gelatin alone.

For film thickness measurements, a small part in the interior of the film area was removed mechanically with a spatula, and the difference between substrate and film level was measured by surface profilometry. ${ }^{17}$ An example of such a profile is displayed in Fig. 1. The film thickness as a function of rotation rate was investigated for a pure gelatin $(20 \mathrm{~g} / \mathrm{l})$ and a $\mathrm{PbS}$-gelatin solution (concentration of gelatin $10 \mathrm{~g} / 1$ and of $\mathrm{PbS} 8.2 \mathrm{~g} / 1$, corresponding to a lead sulfide content in the film of $45 \mathrm{wt} . \%)$. By varying the rotation rate from 300 to $2000 \mathrm{rpm}$, the film thickness decreases from ca. $10 \mu \mathrm{m}$ to ca. $200 \mathrm{~nm}$ for the pure gelatin solutions (Fig. 2); varying the rotation rate from 200 to 3000 rpm decreases the film thickness from ca. $1 \mu \mathrm{m}$ to ca. $40 \mathrm{~nm}$ when $\mathrm{PbS}$ is present (Fig. 2). It is obvious that the addition of $\mathrm{PbS}$ reduces the film thickness. It has been reported that

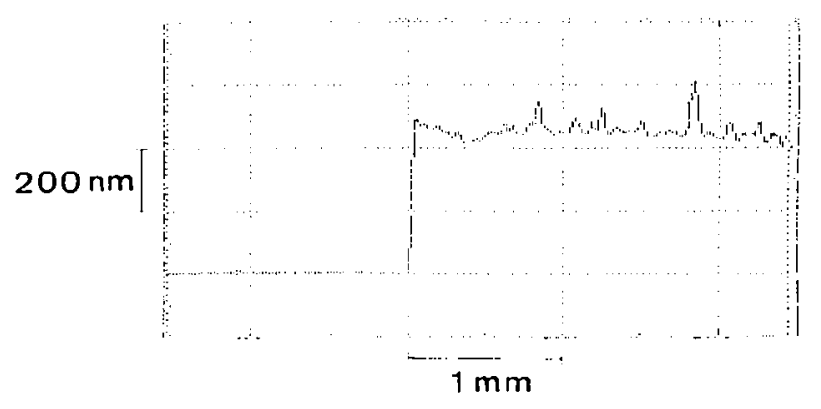

FIG. 1. Step height profile indicating the film thickness for a spin-coated $\mathrm{PbS}$-gelatin nanocomposite containing $45 \mathrm{wt}$ \% $\mathrm{PbS}$. 


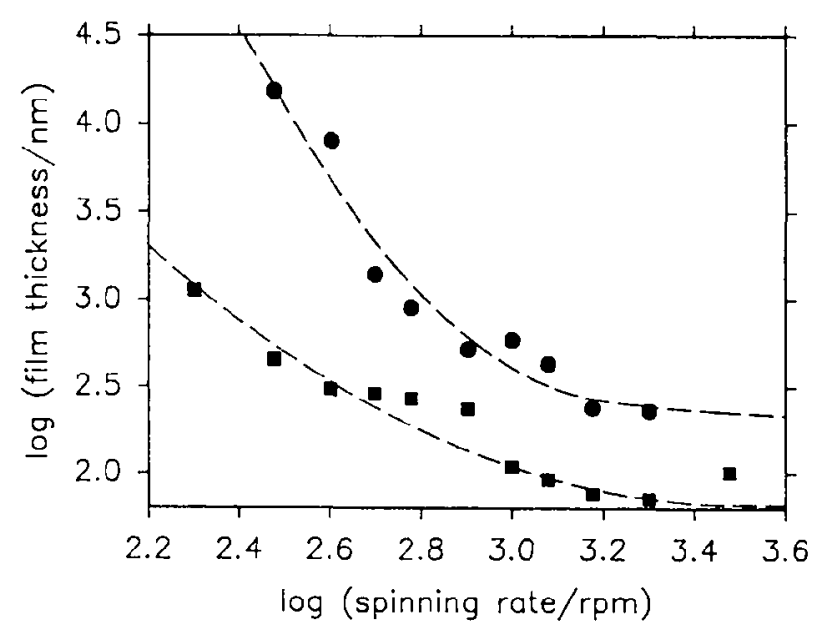

FIG. 2. Film thickness of spin-coated films as a function of the decadic logarithm of the spinning rate (dashed lines are drawn arbi-

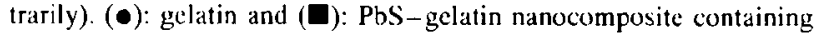
$45 \mathrm{wt}$ \% PbS. Dashed lines are arbitrary.

double-logarithmic plots yield a straight line relationship between film thickness and rotation rate for polymers ${ }^{17}$ and polymer-dye films, ${ }^{22}$ but other reports disagree. ${ }^{15}$ The films described here do not disclose such a linear dependence (Fig. 2).

The surface roughness of the above films was andlyzed by surface profilometry for a film with $45 \mathrm{wt} \%$ $\mathrm{PbS}$. The ratio of surface roughness and film thickness (relative roughness) gives information on the film quality. In the following, the "average roughness" is defined as the average deviation from the mean film level, and the "peak-to-valley roughness" as half the difference between the highest and the lowest detected vertical point. Both types of relative surface roughnesses are displayed over a distance of $80 \mu \mathrm{m}$ and $5 \mathrm{~mm}$ in Figs. 3(a) and 3(b). By necessity, the relative peak-to-valley roughness is larger than the relative average roughness. The relative roughnesses measured over longer distances are larger than those for shorter distances, indicating that there is roughness on a characteristic wavelength on the order of the smaller scanning distance or larger. The relative average roughness docs not exceed 0.08 , and the relative peak-to-valley roughness is below 0.5 . Therefore, no spots were detected on which the substrate is not covered by film.

No significant difference could be detected by scanning electron microscopy (SEM) before and after annealing. Pictures at different magnifications are displayed in Figs. 4(a)-4(c) (86.4 wt. \% PbS). A stereo image [Fig. 4(b)] discloses a surface roughness, as evidenced already by surface profilometry. However, no microphase separation and no objects above ca. $100 \mathrm{~nm}$ are visible. It is not known from these pictures whether the small, often spherical, particles are single $\mathrm{PbS}$

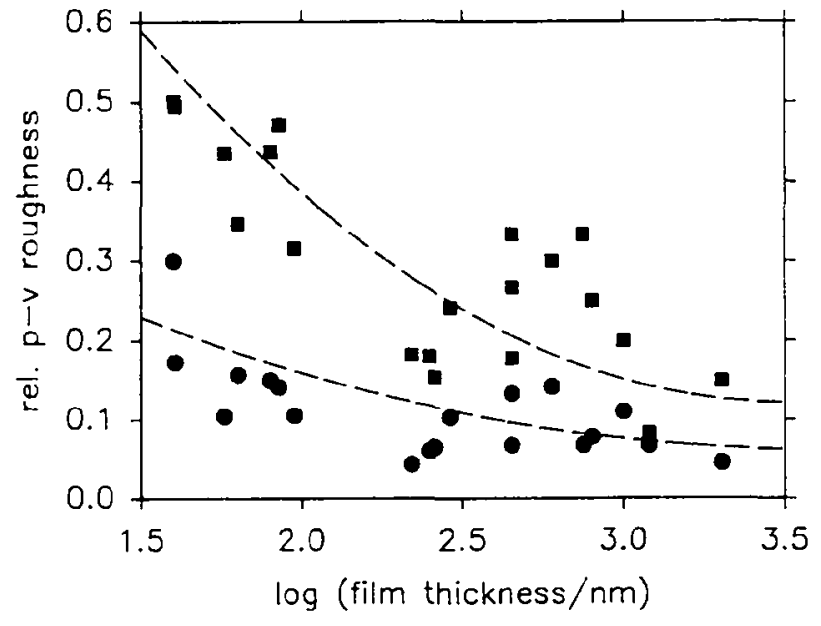

(a)

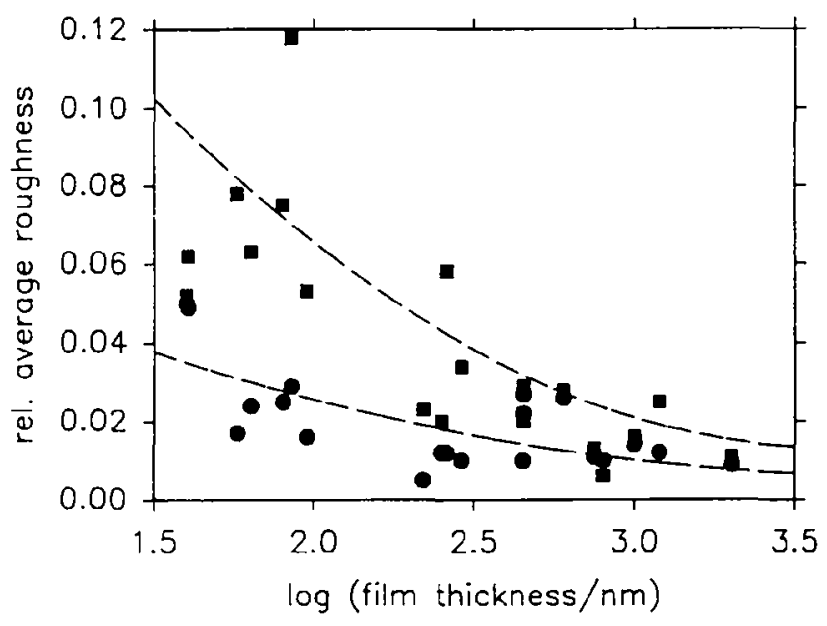

(b)

FIG. 3. Relative peak-to-valley (a) and average surface roughness (b) as a function of $\log$ film thickness (dashed lines are drawn arbitrarily) of $\mathrm{PbS}$-gelatin nanocomposites containing $45 \mathrm{wt}$ \% $\mathrm{PbS}$ measured over a horizontal distance of $5 \mathrm{~mm}(\mathbf{0})$ and $80 \mu \mathrm{m}(\bullet)$.

particles, possibly enveloped by gelatin, or whether these objects are aggregates of smaller particles.

No evidence for large-scale heterogeneitics was observed by transmission electron microscopy (TEM). Figure 4(d) shows a micrograph of a nanocomposite with 68 wt. \% PbS after heat treatment. The bright stripes on both sides of the film cross section represent the embedding material (see Sec. II). Particles with typical dimensions of $2-5 \mathrm{~nm}$ were detected. The particles are crystalline by elcctron diffraction, and the diffraction pattern agrees with a reference of crystalline PbS.

\section{B. Refractive indices}

The refractive index at $632.8 \mathrm{~nm}$ was measured before and after annealing of the films (at $164{ }^{\circ} \mathrm{C}$ and 

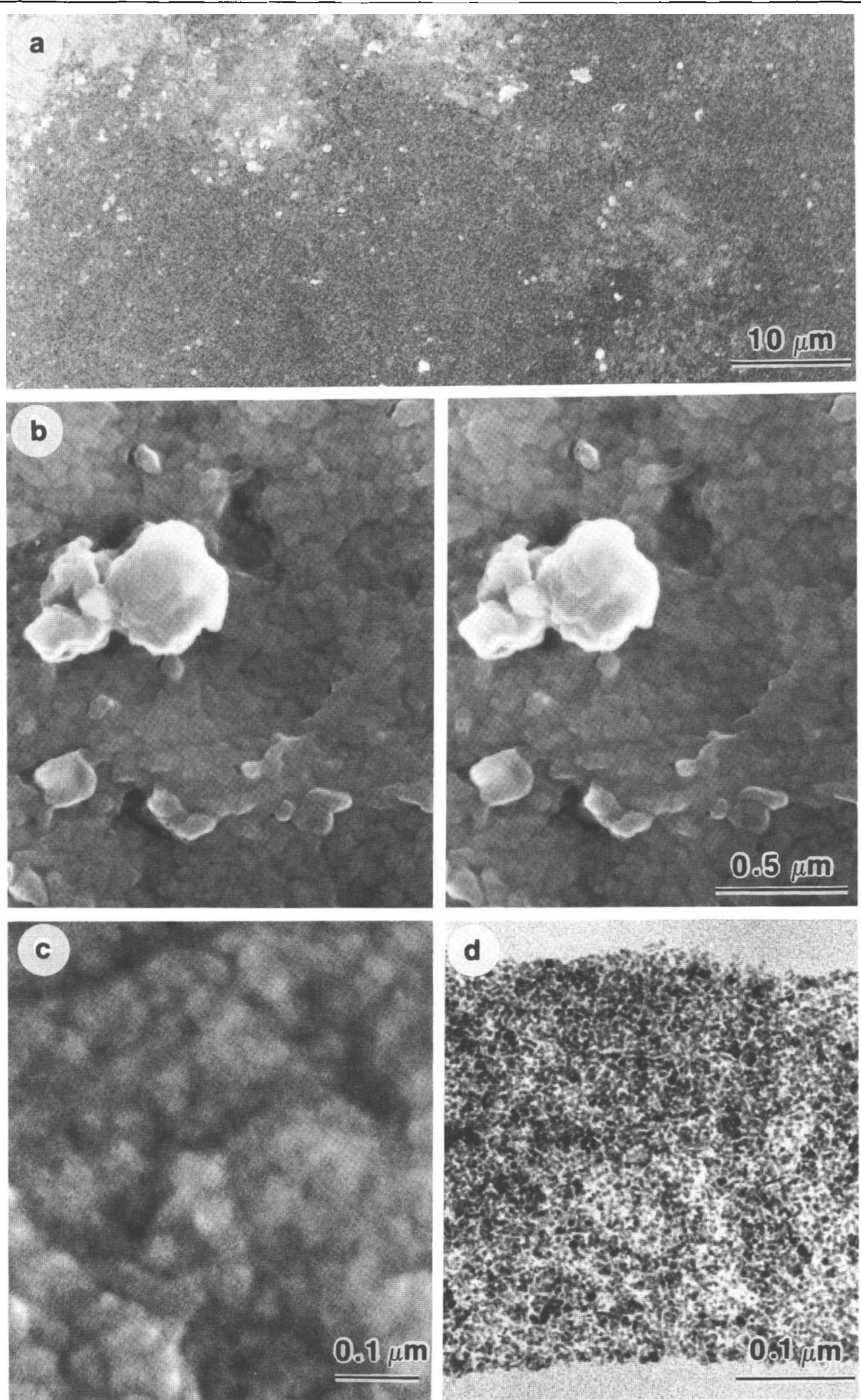

FIG. 4. Electron micrographs of spin-coated PbS-gelatin films. (a)-(c) SEM nicrographs of a film containing 86.4 wt. \% PbS (the white elevation in the stereo micrograph (b) is not representative of the film, but it helps in getting the stereo impression when it is fixed) and (d) TEM micrograph of a film containing $68 \mathrm{wt} . \% \mathrm{PbS}$. 
100 Torr for $2 \mathrm{~h}$ ). The refractive index of films containing 30-86 wt. \% PbS lies in the interval of $1.55-2.50$, increasing with increasing $\mathrm{PbS}$ fraction. It increases upon annealing by ca. $0.02-0.18$, or ca. $1-8 \%$, and the scattering of the refractive index measurements becomes smaller upon annealing. Although the highest absolute and relative enhancements upon annealing are preferentially found for high PbS loadings (>65 wt. \% $\mathrm{PbS}$ ), there is no systematic trend observable. The water content is similar before and after annealing. Thus, if the films lost water during the annealing procedure, they would take on about the same amount again after cooling and exposing to the atmosphere. However, measurements of thickness and surface roughness reveal that the films shrink typically by $5-8 \%$, while the average surface roughness decreases typically by $10-20 \%$, measured over a horizontal distance of $5 \mathrm{~mm}$. Thickness variation upon annealing has also been reported for spin-coated polymer films. ${ }^{20}$ Thus, it seems that the spincoating process leads to a "frozen-in" film structure. The driving force for the film shrinking might be a rearrangement of the morphology of gelatin (changing the density and, hence, the refractive index of gelatin) or a disappcarance of small holes (increasing the volume fraction of the solid components) upon annealing. It scems less likely that the PbS particles change upon annealing.

The refractive index of annealed films as a function of the $\mathrm{PbS}$-gelatin weight and volume ratio is shown in Figs. 5 and 6 . The volume ratios were calculated from the weight ratios using densities of $\rho_{\mathrm{G}}=1.35 \mathrm{~g} / \mathrm{cm}^{3}$ for gelatin $^{24}$ and $\rho_{\mathrm{PbS}}=7.5 \mathrm{~g} / \mathrm{cm}^{3}$ for $\mathrm{PbS}{ }^{25}$ The refractive index appears to depend roughly linearly on the $\mathrm{PbS}$ volume fraction. Corresponding curves, based on a fitting of the equation,

$$
n=\phi_{\mathrm{PbS}} n_{\mathrm{PbS}}+\left(1-\phi_{\mathrm{PbS}}\right) n_{\mathrm{G}},
$$

are also drawn in Figs. 5 and 6, where $n$ is the measured refractive index of the nanocomposite, $n_{\mathrm{G}}$ is the refractive index of gelatin (to be calculated by the regression analysis), $n_{\mathrm{PbS}}$ is the refractive index of the $\mathrm{PbS}$ colloid (to be calculated by the regression analysis), and $\phi_{\mathrm{PbS}}$ is the volume fraction of $\mathrm{PbS}$ colloid. When plotted against weight fraction $\mathrm{PbS}, w_{\mathrm{PbS}}$, the line becomes, of course, a curve of shape,

$$
n=\frac{\rho_{\mathrm{PbS}} n_{\mathrm{G}}-w_{\mathrm{PbS}}\left(\rho_{\mathrm{PbS}} n_{\mathrm{G}}-\rho_{\mathrm{G}} n_{\mathrm{PbS}}\right)}{\rho_{\mathrm{PbS}}-w_{\mathrm{PbS}}\left(\rho_{\mathrm{PbS}}-\rho_{\mathrm{G}}\right)} .
$$

The error limits of the single refractive index values are typically \pm 0.01 to \pm 0.05 (95\% confidence level) for films with a $\mathrm{PbS}$ content below ca. $70 \mathrm{wt} \%$, where the refractive index is ca. 2 . Above $70 \mathrm{wt} \%$ $\mathrm{PbS}$, the error limits increasc significantly, due to the difficulties in the preparation of the related films. For

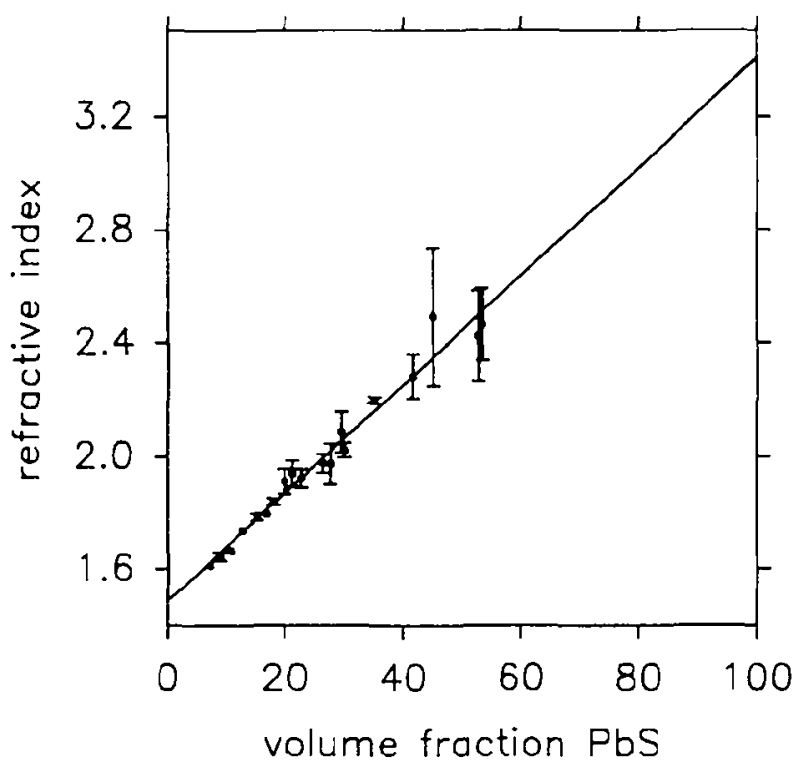

FIG. 5. Refractive index of $\mathrm{PbS}$-gelatin nanocomposites as a function of wcight fractions of $\mathrm{PhS}$. Error bars are related to a $95 \%$ confidence level (visible only for deviations larger than the symbol dimensions). The straight line represents a best-fitting Eq. (1) in the text.

instance, the highest refractive index measured was $2.492 \pm 0.244$ (82.0 wt. \% PbS), followed by $2.465 \pm$ 0.128 (86.4 wt. \% PbS).

The regression analysis leads to a value of $1.49 \pm$ 0.05 (95\% confidence level) for the extrapolation to 0 vol. $\% \mathrm{PbS}$ and of $3.4 \pm 0.1$ (95\% confidence level) for 100 vol. $\%$ PbS. These values should correspond

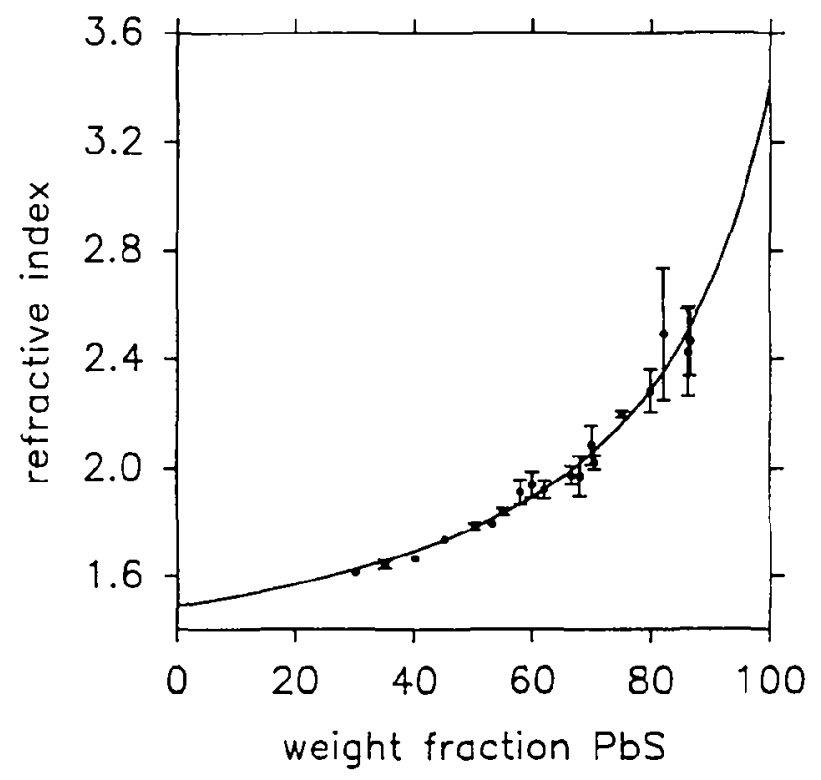

FIG. 6. Refractive index of $\mathrm{PbS}$-gelatin nanocomposites as a function of volume fractions of PbS. Error bars are related to a $95 \%$ confidence level (visible only for deviations larger than the symbol dimonsions). The solid line represents a best-fiting Eq. (2) in the text. 
to the refractive index of "native" gelatin and of colloidal PbS. Indeed, the refractive index for "native" gelatin films is $1.537 \pm 0.008$ ( $95 \%$ confidence level), in good agreement with the literature $\left(1.54^{24}\right)$, while the refractive index of colloidal $\mathrm{PbS}$ is not known, to our knowledge. The refractive index for PbS single crystals is ca. 4.3 at $632.8 \mathrm{~nm}{ }^{1.3}$ The difference between the refractive index of $\mathrm{PbS}$ single crystals and the value obtained by linear regression can hardly be explained by an error in the density used for the calculation of the volume fractions, since a change in the densitics of $\pm 20 \%$ would not change the results significantly. The difference could be interpreted by other effects. The particles might be partially amorphous (see above) and therefore exhibit a refractive index different from that of crystalline $\mathrm{PbS}$, the refractive index could be lower for particles with very small size, ${ }^{23,26,27}$ the dependence of the refractive index from the $\mathrm{PbS}$ volume fraction might become nonlincar for high $\mathrm{PbS}$ volume fractions, and the value could be anything greater than 3.2. Also, the particles might be "corroded", c.g., oxidized to $\mathrm{PbSO}_{4}$, at the surface (however, it has been reported that $\mathrm{PbS}$ polymer nanocomposites are extremcly stable at room temperature ${ }^{4}$ ).

In $\mathrm{SiO}_{2}$-poly(methylmethacrylate) ( $\left.\mathrm{SiO}_{2}-\mathrm{PMMA}\right)$ composites, the refractive index depends linearly on the inorganic volume fraction up to $60 \% .^{1,2}$ According to the refractive index of pure $\mathrm{SiO}_{2}$, probably measured with $\mathrm{SiO}_{2}$ of larger dimensions, a nonlinear volume fraction dependence is expected by those authors at higher volume fractions. 1,2 However, the possible effect of a particle size dependence on the refractive index is not mentioned. There are no experimental data at sufficiently high volume fractions for a safe illustration of a nonlinear volume fraction dependence at volume fractions.

\section{ACKNOWLEDGMENTS}

We would like to thank Dr. Paul Walther, Laboratorium für Elcktronenmikroskopic I of the EIHZürich, for assistance in taking electron micrographs, and the Swiss "Nationales Forschungsprogramm 19" for financial support.

\section{REFERENCES}

1. E. J. A. Pope. M. Asami, and J. D. Mackenzie, J. Mater. Res. 4, $1018(1989)$

2. E. J. A. Pope, M. Asami, and J.D. Mackenzie, in Multicomponent Ultrafine Microstructures, edited by L.. E. McCandlish, D. E. Polk, R. W. Sicgel, and (i. II. Kcar (Matcr. Res. Soc. Symp. Proc. 132, Pittsburgh. P'A. 1989), p. 105.

3. P. N. Dunlap and S.F. Howe, Polym. Compos. 12, 39 (1991)

4. W. Mahler, U.S. Patent 4738798 (1988).

5. W. Mahler. Inorg. Chem. 27, 4.35 (1988).

6. Y. Wang. A. Suna, and W. Mahler, in Nomlinear Optical Properties of Polymers, edited by A. J. Hecger, J. Orenstein, and D. R. Ulrich (Mater. Res. Soc. Symp. Proc. 109, Pittsburgh, PA, 1988), p. 187.

7. M. Weibel, W. Cascri, U.W. Suter, H. Kiess, and E. Wchrli, Polym. Adv. 'Technol. 2, 75 (1991).

8. Polymer Handbook, edited by J. Brandrup and F. H. Immergut (John Wiley and Sons, New York, 1989).

9. 'T'. Sugiyama, T. Wada, and H. Sasabc. Synthet. Met. 28. C.323 (1989).

10. H-U. Simmrock, A. Mathy. L. Domingue?, W. H. Meyer, and G. Wegner, Adv. Mater. 1, 294 (1989)

11. R. A. Gaudiana and R. A. Minns, J. Macromol. Sci. A 28. 831 $(1991)$.

12. H. G. Rogers. R. A. Gaudiana, W. C. Hollinsed, P. S. Kalyanaraman. J.S. Manello, C. McGowan, R. A. Minns, and R. Sahatjian, Macromolecules 18, 1058 (1985).

13. Handbook of Optical Constants of Solids, edited by F.. D. Palik (Academic Press, Orlando, FL, 1985).

14. W. H. Westphal, Physik (Springer, Berlin, 1963), p. 507.

15. ^. Weill, Springer Proc. Phys. 13. 51 (1986).

16. J. H. L.uft, J. Biophys. Biochem. Cytol. 9, 409 (1961)

17. K. J. Skrobis, D. D. Denton, and A. V. Skrobis, Polym. Eng. Sci. 30, $193(1990)$.

18. B. Reisfeld, S. G. Bankoff, and S. H. Davis, J. Appl. Phys. 70, 5258 (1991)

19. B. Reisfeld, S. G. Bankoff, and S.H. Davis, J. Appl. Phys. 70, $5267(1991)$.

20. J. Russat. Surf. Interface Anal. 11, 414 (1988).

21. L. M. Peurrung and D. B. Graves, J. Electrochem. Soc. 138, 2115 $(1991)$.

22. K-Y. Law, Polymer 23, 1627 (1982).

23. A.J. Nozik, F. Williams, M.T. Nenadović, T. Rajh, and (O.I. Mićić, J. Phys. Chem. 89, 397 (1985).

24. Incyclopedia of Polymer Science and lingineering, edited by II. F. Mark, N. M. Bikales, C. G. Overberger, (j. Menges, and J. I. Kroschwitz. (John Wiley and Sons, New York, 1987), Vol. 7.

25. Handbook of Chemistry and Physics, edited by R. C. Weast (CRC. Press. Boca Raton. FI. 1985).

26. R. Rossetti, R. Hull. J. M. Gibson. and I.. E. Brus, J. Chem. Phys. 83, 1406 (1985).

27. Y. Wang and N. Herron, J. Phys. Chem. 91, 257 (1987). 